\title{
Kinetics of light-assisted physical ageing in S-rich arsenic sulphide glasses
}

\author{
A KOZDRAS \\ Faculty of Physics, Opole University of Technology, Opole 45370, Poland \\ MS received 23 October 2015; accepted 18 February 2016
}

\begin{abstract}
The obtained results show that kinetics of light-assisted physical ageing in S-rich glasses can be well fitted with stretch-exponential Kohlrausch-type function, in which exponent $\beta$-values and the effective time relaxation constant $\tau$ depend on the wavelength of incident photons. The obtained $\beta$-values exhibit well-expressed minimum for the structural relaxation stimulated by light with energy of quanta comparable with the optical gap of the material. This effect is found to be similar to Se-rich glasses.
\end{abstract}

Keywords. Chalcogenide glass; ageing; calorimetry; relaxation.

\section{Introduction}

Recently, it was shown that kinetics of dark- and light-assisted isothermal physical ageing, expressed through the enthalpy losses $\Delta H(t)$, in Se-based chalcogenide glasses (ChG) can be well fitted with stretch-exponential Kohlrausch-type function $[1,2]$ :

$$
\Delta H(t)=\Delta H^{\max }\left[1-\exp \left(-\frac{t}{\tau}\right)^{\beta}\right],
$$

where $\Delta H^{\max }$ is the maximum enthalpy a glass can lose after infinitely long period of physical ageing, $\tau$ the effective time constant and $\beta$ a so-called Kohlrausch exponent or stretching parameter (also known as non-exponentionality or dispersivity index), ranging between 0 (high-dispersive process) and 1 (single exponential relaxation) $[3,4]$.

In the case of light-assisted physical ageing in selenide $\mathrm{ChG}$, Kohlrausch exponent $\beta$ and effective time relaxation constant $\tau$ are found to be dependent on the wavelength of incident photons and duration of storage [2]. The obtained $\beta$-values for Se-rich $\mathrm{ChG}$ grouped around $3 / 7$ and $1 / 3$ values [2]. The first value was directly predicted by Phillips field-free axiomatic diffusion-to-traps model [5], while the second one $\beta=1 / 3$ required either an assumption on three distinct channels, one of them being effectively relaxational, or the application of field-forced relaxation and fractal models [6]. Slight deviations of the experimentally obtained $\beta$-values from the theoretically predicted ones were associated with additional contribution from other less efficient relaxational channels not accounted by the model [2]. However, all of the above Se-based ChG with non-zero light-assisted physical ageing effect were also characterized by a non-zero effect of dark physical ageing [7], and vice versa-all of the glasses with negligible dark physical ageing effect were also

a.kozdras@po.opole.pl characterized by insignificant light-assisted physical ageing effect [8]. Therefore, coupling between these two effects, as well as the pure contribution of light-assisted structural relaxation into the parameters of Kohlrausch-type function describing the overall physical ageing kinetics, remain an open question. To break the coupling between dark- and light-assisted physical ageing effects one would need to investigate glasses with negligible dark component of physical ageing, but significant light-induced structural relaxation. The best candidate for such investigations can be found among the As-S glasses, because they possess almost $\sim 1$ order higher sensitivity to photoexposure (or other type irradiation) in comparison to selenide glasses [8], As-Se ChG being their typical representatives.

So, in present paper the kinetics of enthalpy losses in $\mathrm{S}$-based ChG under the exposure to the light of different discrete wavelengths at the ambient conditions are studied and compared to the previous data obtained on isomorphic Se-based ChG [2]. The structural relaxation through the glass-to-supercooled liquid transition interval is investigated by conventional differential scanning calorimetry (DSC) technique.

\section{Experimental}

The glasses of $\mathrm{As}_{x} \mathrm{~S}_{100-x}(x=25,30$ and 40) were prepared by conventional melt quenching route in the evacuated quartz ampoules from a mixture of high-purity precursors. The furnace was rocked at the maximum temperature $\left(500-700^{\circ} \mathrm{C}\right.$ depending on the composition) for 10-24 $\mathrm{h}$ to homogenize the melt. The compositions were chosen owing to the glassforming regions of the above $\mathrm{ChG}$ systems and previous data on the kinetics of physical ageing effects in dark and under the influence of various external factors [9-11].

Amorphous state and the composition of as-prepared glasses were controlled visually by a characteristic conchlike fracture, data of X-ray diffraction and X-ray photoelectron 
spectroscopy. Homogeneity of the obtained glasses was inferred from the IR microscope inspection and Raman spectroscopy data. Bulk samples in the form of thick $(\sim 3 \mathrm{~mm})$ plates, prepared for DSC measurements, were used for the investigations. Before the experiment had started, all samples were rejuvenated by heating to $\sim 40 \mathrm{~K}$ above the corresponding onset values of glass transition temperature $\left(T_{\mathrm{g}}\right)$ to erase any thermal prehistory of the samples. The cooling rate during rejuvenation procedure was equal to the heating rate of DSC measurements $\left(5 \mathrm{~K} \mathrm{~min}^{-1}\right)$. Then the identical batches of rejuvenated samples were stored at room temperature $\left(T_{\mathrm{a}} \sim 295 \mathrm{~K}\right)$ in the dark or under the illumination with continuous light of different discrete wavelengths (430, $505,590,620,660,720,780,970 \mathrm{~nm})$ obtained from light emitting diode (LED) sources (Roithner LaserTechnik $\mathrm{GmbH}$, Austria). The width of half maximum of emission lines for each wavelength did not exceed $\sim 50 \mathrm{~nm}$. The power of every LED was chosen in order to keep a constant photon flux $\left(\sim 2.5 \times 10^{16}\right.$ photons $\left.\mathrm{cm}^{-2} \mathrm{~s}^{-1}\right)$ for each wavelength. It was controlled during the whole period of light irradiation by Optical Power Meter PM100A equipped with Thermal Power Head S302C. The irradiation chamber was built in such a way that it allowed temperature control and air convection to prevent substantial heating of the samples during photoexposure.

Samples were measured with conventional DSC routinely after certain periods ( $\leq 60$ days) of storage in the dark or under the light exposure to obtain kinetic curves, assuming that the rejuvenated samples are quite close to the as-prepared ones. The DSC measurements were performed on NETZSCH 404/3/F microcalorimeter pre-calibrated with a set of standard elements, the DSC curves were recorded in the dark (ex situ) at the ambient atmosphere with $5 \mathrm{~K} \mathrm{~min}^{-1}$ heating rate. Same calibration procedure was repeated each time during routine kinetics measurements. Three independent DSC measurements were performed in each case to confirm the reproducibility of the obtained results. DSC curves of the rejuvenated samples were compared each time after the ageing test and no essential differences were obtained between them. In particular, the value of specific heat capacity jump $\Delta C_{\mathrm{p}}\left(T_{\mathrm{g}}\right)$ did not change for a given composition neither after the illumination nor after the dark storage and the initial value of $T_{\mathrm{g}}$ was recovered after each rejuvenation, which confirms that the glass had undergone only physical ageing, eliminating any crystallization or phase separation effects from consideration. Raw DSC data were processed using Netzsch PROTEUS ANALYSIS ${ }^{\circledR}$ PC software package.

\section{Results and discussion}

Typical DSC curves recorded for $\mathrm{As}_{25} \mathrm{~S}_{75}, \mathrm{As}_{30} \mathrm{~S}_{70}$ and $\mathrm{As}_{40} \mathrm{~S}_{60}$ glasses after equal time intervals of dark storage and illumination with light of different discrete wavelengths are shown in figure 1. Compared to dark, significant
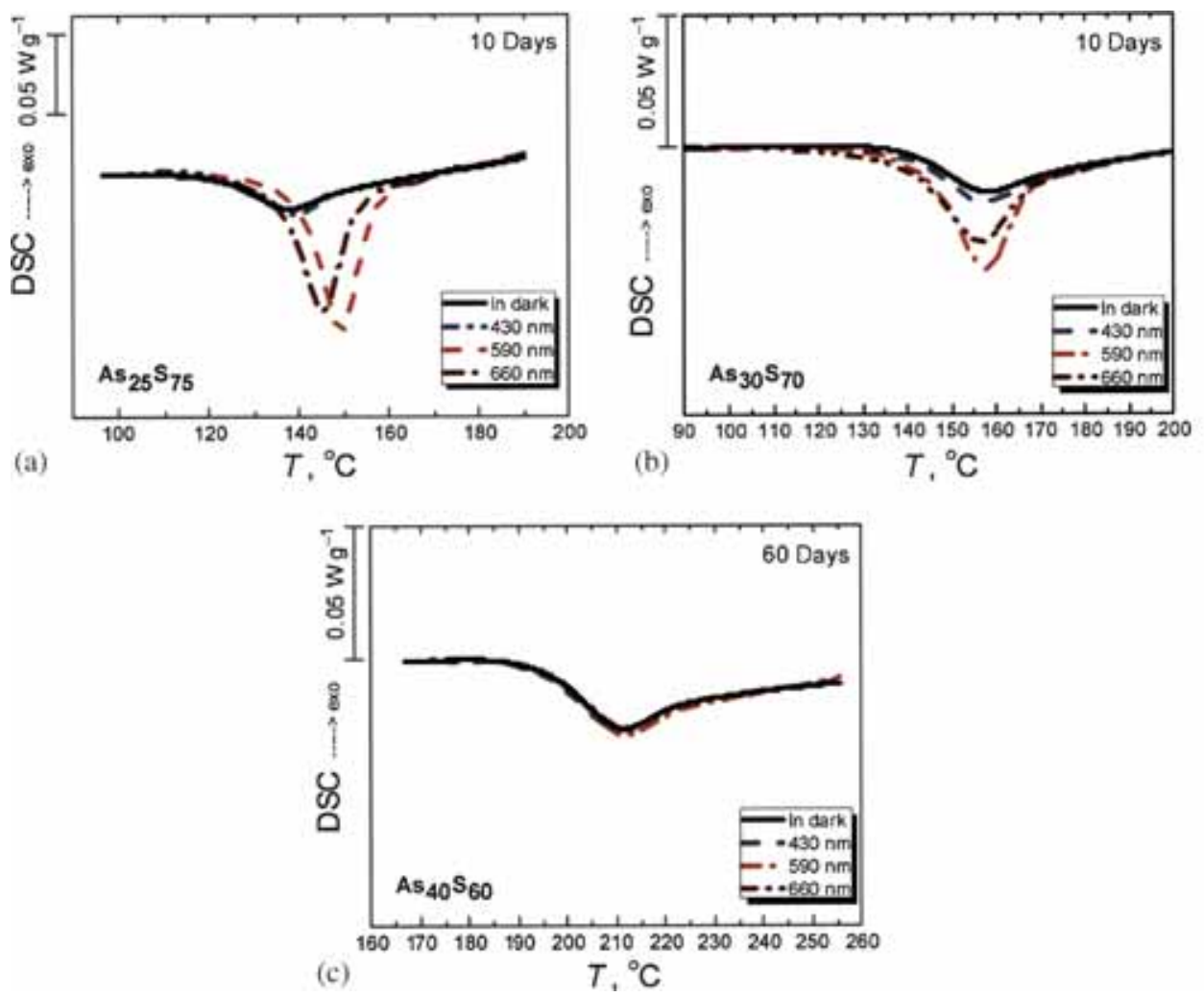

Figure 1. Typical DSC curves of (a) $\mathrm{As}_{25} \mathrm{Se}_{75}$, (b) $\mathrm{As}_{30} \mathrm{~S}_{70}$ and (c) $\mathrm{As}_{40} \mathrm{~S}_{60}$ samples recorded after 10 or 60 days of storage in the dark or under the exposure to the light of different wavelengths. 
light-assisted physical ageing is recorded in $\mathrm{As}_{25} \mathrm{~S}_{75}$ and $\mathrm{As}_{30} \mathrm{~S}_{70}$ glasses under photoexposure with certain wavelengths (figure 1a and b), while negligible changes after 60 days (maximum period used) of photoexposure are observed in DSC curves of $\mathrm{As}_{40} \mathrm{~S}_{60}$ glass (figure 1c). Light-assisted physical ageing in $\mathrm{As}_{25} \mathrm{~S}_{75}$ and $\mathrm{As}_{30} \mathrm{~S}_{70}$ glasses is revealed by DSC as development of strong endothermic peak superimposed on the endothermic step of glass transition signal and its displacement towards higher temperatures (figure 1a and b), similar to selenide $\mathrm{ChG}[1,7,11]$. However, the magnitude of light-assisted physical ageing effect in arsenic sulphide glasses is $\sim 1$ order larger than analogous changes in arsenic selenide glasses of same compositions (compare $\mathrm{As}_{30} \mathrm{Se}_{70}$ and $\mathrm{As}_{30} \mathrm{~S}_{70}$, for example [8]). This difference was attributed earlier to a significant role of valence alternation pairs (VAPs) [8,12], which formation is known to be more efficient in sulphides than in selenides (magnitude of the induced darkening effect associated with VAPs formation decreases in the row $\mathrm{S} \rightarrow \mathrm{Se} \rightarrow \mathrm{Te}$ ) [13]. The difference in the integrated areas under the DSC signal of aged and rejuvenated (non-aged) $\mathrm{ChG}$ is directly proportional to the enthalpy losses $\Delta H^{1}$, which is used in further analysis of ageing kinetics.

Kinetics of enthalpy losses $\Delta H(t)$ for S-rich glasses caused by their dark storage and exposure to a light of different discrete wavelengths are shown in figure 2 for $\mathrm{As}_{25} \mathrm{~S}_{75}$ and $\mathrm{As}_{30} \mathrm{~S}_{70}$ glasses. It is clearly seen that dark physical ageing component (caused by 60 days period of storage in the dark at room temperature) in these $\mathrm{ChG}$ is negligible. On the other hand, maximum light-assisted physical ageing is achieved by the exposure to the light with energy of the photons comparable to the optical gap of $\mathrm{As}_{25} \mathrm{~S}_{75}$ and $\mathrm{As}_{30} \mathrm{~S}_{70}$ glasses [8].

If thermal contribution is excluded, which is shown to be the case for this kind of experiment earlier [2], the observed $\Delta H(t)$ kinetics can be related exclusively to the lightassisted structural perturbation within the glass network. Fitting of $\Delta H(t)$ kinetics shown in figure 2 to the equation (1) was performed in OriginPro(c) software with amplitude, $\beta$ and $\tau$ parameters freed. The iterative Levenberge-Marquardt method of nonlinear least-squares minimization was used to obtain the best fit to the experimental curve. The initial conditions used to start the fitting procedure were identical for each kinetic curve, being $1 \mathrm{~J} \mathrm{~g}^{-1}, 0.5$ and 5 days for amplitude, $\beta$ and $\tau$ parameters, respectively. The fitting shows that $\beta$ and $\tau$ exhibit a resonant-like behaviour (figure 2) within the range of expected optical gap $E_{\mathrm{g}}$-values $[9,10]$, similar to the results obtained earlier for Se-rich $\mathrm{ChG}$ [2]. It can be seen that obtained $\beta$-values for the S-rich $\mathrm{ChG}$ subjected to closeto-bandgap irradiation is definitely lower than the value $3 / 5$ or $3 / 7$, predicted by Phillips fields-free axiomatic diffusionto-traps model [5]. Within this model, the dimensionless
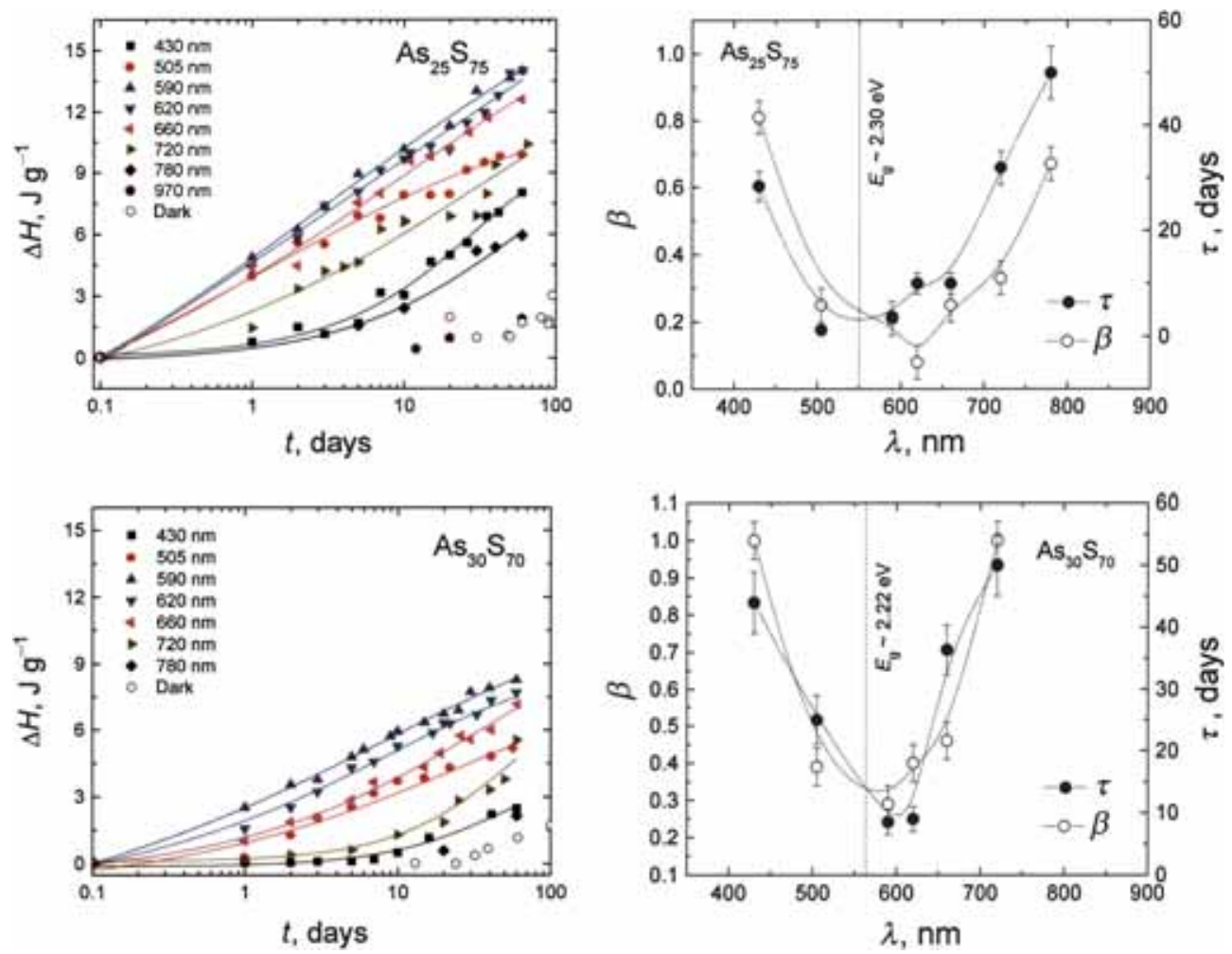

Figure 2. (Colour online) Left panel: $\Delta H(t)$ kinetics of light-assisted physical ageing in S-rich $\mathrm{As}_{x} \mathrm{~S}_{100-x}$ glasses and their best fits (solid lines); right panel: spectral dependence of fit parameters as obtained from fitting $\Delta H(t)$ curves with function (1). The dotted lines are drawn as guide to the eyes. Optical gap $E_{\mathrm{g}}$-values are taken from the study by Feltz [9] and Borisova [10]. 
fractional parameter $\beta$ is considered as topological parameter dependent on the effective dimensionality $d^{*}$ of the configuration space, in which the excitation diffuses, and is given by $\beta=d^{*} /\left(d^{*}+2\right)$ [5]. The effective dimensionality in the diffusion-to-traps universal minimalist model is defined as $d^{*}=f d$, where $d$ is the dimension of Cartesian scattering space and $f$ the fraction of channels activated for the particular relaxation process. In the simple case of $d=3$ with all channels activated ( $f=1$ ), a stretching exponent of $\beta=3 / 5$ is obtained [5]. If the equipartitioning of the relaxation channels into short- and long-range contributions can be assumed and only one channel is effective (the other being cyclical, ineffective or minor), then $\beta=3 / 7$ is obtained owing to $f=1 / 2$. It is shown to be the case for short-term physical ageing of Se-based ChG [2].

In the case of the investigated S-rich $\mathrm{ChG}$ exposed to the close-to-bandgap light, fractional parameter $\beta$ approaches $1 / 3$ value, indicating that a fraction of channels activated for this relaxation process should be close to $f=1 / 3$ if diffusion-to-traps model applies. Following the above formalism, we should either assume that light activates three channels, only one of them being relaxationally effective, or topological dependence of $\beta$ on the effective dimensionality $d^{*}$ is different in the presence of external fields (caused by photoexposure) and in the case of field-free structural relaxation. In the case of external field, $\beta=1 / 3$ value can be directly predicted by field-forced relaxation fractal model $[6,14]$. Because dark physical ageing is miniscule in $\mathrm{As}_{25} \mathrm{~S}_{75}$ and $\mathrm{As}_{30} \mathrm{~S}_{70}$ samples during first 60 days, the channels responsible for light-assisted structural relaxation in these glasses should be exclusively related to the interaction with light. Let us consider a possible physical origin of such channels.

When the energy of incident photons approaches bandgap value the probability of VAPs formation increases drastically, which could be well inferred from a spectral dependence of photo-darkening $[13,15]$ relying mostly on these defects. The VAPs usually appear as pairs of under-coordinated negatively charged and over-coordinated positively charged diamagnetic defects accompanied by structural rearrangements at the medium-range ordering of glasses [12,16]. Because photoexposure occurs from surface to bulk, there should be a gradient of VAPs concentration and generated charge carriers (holes and electrons) through the sample. Therefore, diffusion of these species towards bulk should occur, which together with the external electric fields of charged VAPs would decrease the effective dimensionality $d^{*}$ for the relaxation process, as predicted by the field-forced relaxation fractal model [6]. Then, $\beta \sim 1 / 3$ value can be achieved as a result of field-forced relaxation theory $[6,15]$. Because dark physical ageing is negligible in $\mathrm{As}_{25} \mathrm{~S}_{75}$ and $\mathrm{As}_{30} \mathrm{~S}_{70}$ glasses (figure 2), $\beta \sim 1 / 3$ purely corresponds to structural relaxation caused by close-to-bandgap light.

\section{Conclusions}

So, contrary to structural relaxation during short-term dark physical ageing, where $\beta \sim 3 / 7$ corresponds to the value predicted by the Phillips field-free diffusion-to-traps model when one of two relaxation channels is activated and effective, the structural relaxation during light-assisted physical ageing occurs with the reduced effective dimensions, leading to $\beta$-values close to $\sim 1 / 3$ in agreement with the Phillips field-forced diffusion-to-traps model. The decrease in effective space dimensionality is assumed to be caused by inequilibrium concentration of VAPs and transient charge carriers generated by close-to-bandgap light.

\section{Acknowledgements}

I thank Dr Roman Golovchak, Austin Peay State University (Clarksville, TN), for valuable discussions and Prof Mihail Iovu, Institute of Applied Physics (Academy of Sciences of Moldova), for kindly provided samples.

\section{References}

[1] Golovchak R, Kozdras A, Balitska V and Shpotyuk O 2012 J. Phys.: Condens. Matter 24505106

[2] Golovchak R, Kozdras A and Shpotyuk O 2014 J. Mat. Sci. 492844

[3] Angell C A, Ngai K L, McKenna G B, McMillan P F and Martin S W 2000 J. Appl. Phys. 883113

[4] Ngai K L 2011 Relaxation and diffusion in complex systems (New York-Dordrecht-Heidelberg-London: Springer) p 835

[5] Phillips J C 1996 Rep. Prog. Phys. 591133

[6] Macdonald J R and Phillips J C 2005 J. Chem. Phys. 122074510

[7] Golovchak R, Gorecki Cz, Kozdras A and Shpotyuk O 2006 Solid State Commun. 13767

[8] Kozdras A, Golovchak R, Shpotyuk O, Szymura S, Saiter A and Saiter J-M 2011 J. Mat. Res. 262420

[9] Feltz A 1993 Amorphous inorganic materials and glasses (Wiley-VCH: Weinheim) p 448

[10] Borisova Z U 1981 Glassy semiconductors (New York: Plenum Press) p 344

[11] Shpotyuk O, Golovchak R and Kozdras A 2014 Chalcogenide glasses: preparation, properties and application J-L Adam, $\mathrm{X}$ Zhang (eds) (Cambridge: Woodhead Publishing) p 209

[12] Mott N F and Davis E A 1971 Electronic processes in noncrystalline materials (Oxford: Clarendon Press) p 437

[13] Kolobov A V (ed) 2003 Photo-induced metastability in amorphous semiconductors (Weinheim: Wiley-VCH) p 436

[14] Naumis G G and Phillips J C 2012 J. Non-Cryst. Solids 358893

[15] Tanaka K 1986 Japanese J. Appl. Phys. 25779

[16] Kastner M, Adler D and Fritzsche H 1976 Phys. Rev. Lett. 371504 